\title{
Differences in complexity of glycemic profile in survivors and nonsurvivors in an intensive care unit: A pilot study*
}

\author{
Krista Lundelin, PhD; Luis Vigil, MD; Susana Bua, MD; Ivan Gomez-Mestre, PhD; Teresa Honrubia, MD; \\ Manuel Varela, PhD
}

Objective: To investigate glycemic dynamics and its relation with mortality in critically ill patients. We searched for differences in complexity of the glycemic profile between survivors and nonsurvivors in patients admitted to a multidisciplinary intensive care unit.

Design: Prospective, observational study, convenience sample.

Settings: Multidisciplinary intensive care unit of a teaching hospital in Madrid, Spain.

Patients: A convenience sample of 42 patients, aged 29 to 86 yrs, admitted to an intensive care unit with an Acute Physiology and Chronic Health Evaluation II score of $>14$ and with an anticipated intensive care unit stay of $>72 \mathrm{hrs}$.

Interventions: A continuous glucose monitoring system was used to measure subcutaneous interstitial fluid glucose levels every $\mathbf{5}$ mins for $\mathbf{4 8}$ hrs during the first days of intensive care unit stay. A 24-hr period ( $n=288$ measurements) was used as time series for complexity analysis of the glycemic profile.

Measurements: Complexity of the glycemic profile was evaluated by means of detrended fluctuation analysis. Other conventional measurements of variability (range, SD, and Mean Amplitude of Glycemic Excursions) were also calculated.
Main Results: Ten patients died during their intensive care unit stay. Glycemic profile was significantly more complex (lower detrended fluctuation analysis) in survivors (mean detrended fluctuation analysis, 1.49; 95\% confidence interval, $1.44-1.53)$ than in nonsurvivors $(1.60 ; 95 \%$ confidence interval, 1.52-1.68). This difference persisted after accounting for the presence of diabetes. In a logistic regression model, the odds ratio for death was 2.18 for every 0.1 change in detrended fluctuation analysis.

Age, gender, Simplified Acute Physiologic Score 3 or Acute Physiologic and Chronic Health Evaluation II scores failed to explain differences in survivorship. Conventional variability measurements did not differ between survivors and nonsurvivors.

Conclusions: Complexity of the glycemic profile of critically ill patients varies significantly between survivors and nonsurvivors. Loss of complexity in glycemia time series, evaluated by detrended fluctuation analysis, is associated with higher mortality. (Crit Care Med 2010; 38:849-854)

KEY WORDS: critical illness; intensive care unit; glucose; glucometry; detrended fluctuation analysis; nonlinear dynamics
I $\mathrm{n}$ recent years, there has been a growing interest in hyperglycemia associated with critical illness. Hyperglycemia in critically ill patients is a consequence of several factors, including increased cortisol, catecholamines, glucagon, growth hormone, gluconeogenesis, and glycogenolysis $(1,2)$. In addition, insulin resistance has been demonstrated in $>80 \%$ of critically ill patients (3).

There is a wealth of observational evidence from different, mainly surgi-

*See also p. 996.

From the Servicio de Medicina Interna (KL, LV, MV) and Servicio de Medicina Intensiva (SB, TH), Hospital de Mostoles, Madrid, Spain; and Estación Biológica de Doñana (IG-M), CSIC, Madrid, Spain.

The authors have not disclosed any potential conflicts of interest.

For information regarding this article, E-mail: mvarela.hmtl@salud.madrid.org

Copyright (C) 2010 by the Society of Critical Care Medicine and Lippincott Williams \& Wilkins cal, patient populations demonstrating that hyperglycemia is associated with poor clinical outcomes in critically ill patients (4-6). An important limitation in such observational evidence is that it cannot prove that hyperglycemia causes poor clinical outcomes; hyperglycemia may merely be a marker of severe illness.

There is evidence from some randomized trials that correction and prevention of hyperglycemia improve morbidity and may also decrease mortality in some critically ill patients $(7,8)$. This suggests that there is a causal relationship between hyperglycemia and poor outcomes. Nevertheless, the optimal target blood glucose is controversial, and a widely accepted insulin regimen has not been established. Furthermore, other intervention studies have yielded contradictory results (9).

In recent studies, variability in blood glucose levels has emerged as a new predictor of mortality in intensive care unit
(ICU) patients $(10,11)$. In the retrospective studies by Egi et al (10) and Krinsley (11), the glycemic variability was expressed as the SD of each patient's blood glucose levels extracted from electronically stored biochemical databases. The authors proposed that glycemic variability should be added as a metric to analyze ongoing and future clinical trials on intensive insulin therapy.

We hypothesized that new techniques derived from nonlinear dynamics and fractal geometry could offer a more indepth view of the glucoregulatory process than the classic glucose variability measures (SD or Mean Amplitude of Glycemic Excursions) (12), therefore allowing for the detection of slighter changes arguably correlated with the patient's physiologic status.

Nonlineal dynamics (the study of the behavior of nonlineal deterministic systems) is increasingly been used in physiologic studies. Nonlineal systems display an extremely complex output that, 
although being rigorously deterministic, is unpredictable and, at first glance, seems to be random (thus, the term "pseudorandom"). These systems have some striking similarities with certain physiologic mechanisms: They exhibit a pseudorandom behavior, they tend to develop spontaneous rhythms, and most notably, they have a strong tendency to remain in a narrow range of values ("strange attractors"), displaying a behavior that could easily be called homeostatic.

Complexity analysis of time series has been widely used in the study of variability of biological phenomena, such as cardiac interbeat interval (13), cardiac arrhythmia $(14,15)$, intracranial hypertension (16), sepsis and organ failure $(17,18)$, temperature $(19,20)$ and electroencephalogram activity (21, 22). Ogata et al (23) and Churruca et al (24) have analyzed diabetes-related alterations of glucose control by means of complexity analysis of the glycemic profile. They have reported diminished complexity of glycemic profile in diabetic patients vs. healthy volunteers. Several authors have proposed that critical illness and multisystem organ dysfunction are characterized by the phenomenon of decomplexification (25). Healthy state exhibits some degree of (pseudo)random variability in physiologic variables, such as heart rate or temperature. Loss of such irregularity (and consequently of complexity) is one of the hallmarks of critical illness (26, 27).

It should be noted that complexity and variability, although seemingly related, are quite different and often contradictory concepts. A key difference between variability and complexity metrics is that, although variability is based on conventional statistics (range, SD) and, thus, takes each measurement as an independent value, in complexity analysis, each measure is related to its neighbors. This arguably allows complexity analysis to detect minor systemic dysfunctions, not perceived by variability studies. In general, a healthy regulatory system displays a complex output, with frequent and quick corrections of even small deviations. On the other hand, a failing regulatory system will be sluggish and allow for greater deviations before reacting. Therefore, as a rule of thumb, healthy systems have a high complexity and low variability, while failing systems display lower complexity and higher variability.
The aim of this pilot study was to investigate the complexity of the glycemic profile in critically ill patients. Namely, we searched for differences in complexity of glycemic profile between the patients who survive and the patients who die in the ICU. We hypothesized that the glycemic profile would be less complex in nonsurvivors.

\section{MATERIALS AND METHODS}

\section{Patients}

A convenience sample of 42 successive patients who were admitted to the ICU of Mostoles Hospital in Madrid, Spain, from November 2007 to January 2009, participated in the study. We included in our study patients aged Š18 yrs, with an Acute Physiology and Chronic Health Evaluation (APACHE) II score of Š14, and with an anticipated ICU stay of $>72$ hrs.

Collected demographic data included age, comorbilities, and reason for intensive care. We also recorded APACHE II score during the first 24 hrs, Simplified Acute Physiologic Score (SAPS) 3 score, blood glucose, and basic hematologic and biochemical parameters at the time of admission. Type of nutrition, units of insulin administered, and use of vasoactive drugs during the registry were also recorded. A patient was considered to have diabetes if he/she had been informed of this diagnosis and received treatment (diet, tablets, or insulin). For the final analysis, we classified the patients as either survivors (those who were finally discharged from the ICU) or nonsurvivors (patients who died in the ICU). Table 1 summarizes the main clinical data.

The study was approved by the institutional ethics committee at Mostoles Hospital (Madrid, Spain), and all subjects, or their legal surrogate, gave their informed consent to participate.

\section{Glycemia Measurements}

A continuous glucose monitoring system (CGMS) (CGMS System Gold, Medtronic MiniMed, Northridge, CA) was used to monitor subcutaneous interstitial fluid glucose levels $(28,29)$. The patients had a sensor inserted in the abdominal subcutaneous tissue, and interstitial glucose was recorded every 5 mins for at least $48 \mathrm{hrs}$ during the first days of their ICU stay. In two patients, for technical reasons, the glycemic recording was not performed until the second and third week. Four finger-stick blood glucose measurements were introduced to the Medtronic MiniMed daily to calibrate the measurements. Otherwise, the patients re-
Table 1. Characteristics of patients included in the study

\begin{tabular}{lc}
\hline \multicolumn{1}{c}{ Characteristic } & $\mathrm{n}=38$ \\
\hline Age, yrs (range) & $59(29-86)$ \\
Sex, male/female & $25 / 13$ \\
Median APACHE II score at first & $19(16-22)$ \\
$\quad$ 24 hrs (IQR) & $61(12)$ \\
Mean SAPS 3 score (SD) & $23(21)$ \\
Mean length of stay, days (SD) & $11(29)$ \\
History of DM type 2, n (\%) & 3 \\
Treated with insulin, n & 8 \\
Treated with oral antidiabetic & \\
$\quad$ agent, diet or both, n & \\
Reason for intensive care, $\mathrm{n}$ & 21 \\
$\quad$ Acute respiratory failure & 4 \\
Gastrointestinal bleeding & 4 \\
Myocardial infarction & 2 \\
Heart failure & 2 \\
Cerebral infarction & 2 \\
Peritonitis & 1 \\
Pancreatitis & 1 \\
Acute renal failure & 1 \\
Trauma & \\
Glucose at admission, mg/dL (sD) & $165(80)$ \\
Location from which the patient & $12 / 23 / 3$ \\
$\quad$ was admitted to the ICU & \\
$\quad$ ward/emergency room/other & \\
$\quad$ ICU & $10(26)$ \\
Mortality, n (\%) & \\
\hline
\end{tabular}

APACHE, Acute Physiology and Chronic Health Evaluation; IQR, interquartile range; SAPS, Simplified Acute Physiology Score; DM, diabetes mellitus; ICU, intensive care unit.

ceived standard care, including nutrition and insulin therapy, according to their attending physician. A glucose time series was obtained from each subject and downloaded to a computer. We then extracted a 24-hrlong series for study, from 8 am on day 2 to 8 Am on day 3 (in seven patients, the data set did not start at 8 am because of technical problems, but in every case, there were at least $8 \mathrm{hrs}$ of run-in period before the analyzed data set).

Conventional statistics (mean or median, SD, and Mean Amplitude of Glycemic Excursions) were calculated from each time series.

\section{Complexity Analysis}

Complexity was assessed through detrended fluctuation analysis (DFA). An indepth discussion on DFA is beyond the scope of this paper and can be consulted elsewhere (30). Nonetheless, we provide a succinct description of the analytical procedure in the Appendix. DFA is a unitless metric that estimates the degree of long-range correlations within a signal analyzing how the time series and its linear regression diverge as the "time window" considered increases. Intuitively, DFA can be conceived as representing the span of influence of the different 
points in a time series. In a series with high complexity, the influence of each point rapidly fades away, whereas in a "smoother" series, the influence of each point lasts longer. As a rule of thumb, higher complexities are displayed as lower DFA (until a minimum of 0.5 ).

\section{Statistical Analyses}

The complexity of the glycemic profile, measured as DFA, followed a normal distribution as confirmed by a KolmogorovSmirnov test. Differences in DFA between survivors and nonsurvivors were evaluated using analysis of variance. A predictive model of the final outcome based on complexity and clinical variables was built through logistic regression.

All analyses were performed with SPSS software version 12 (SPSS, Inc., Chicago, IL), and $p<.05$ was considered to be statistically significant. Results are reported as mean values and 95\% confidence interval (CI).

\section{RESULTS}

\section{Patients}

Of the 42 patients enrolled in the study, four patients were excluded because of failure of the glucose monitoring system; all four were survivors. Except for a slightly higher mean APACHE II score (28 vs. 20), the missing patients were similar (regarding gender, age, glucose at admission, and SAPS III score) to those reported.

A total of 28 (74\%) patients survived and ten (26\%) died in the ICU. One patient died in the internal medicine ward 16 days after being discharged from the ICU (and, hence, was considered a survivor in our analyses). The rest of the ICU survivors were discharged home after their hospital stay. There were no significant differences between survivors and nonsurvivors in age, APACHE II, or SAPS 3 scores (Table 2). Glycemia at the time of admission was higher in survivors than in nonsurvivors, although it did not reach statistical significance (mean, $178 \mathrm{mg} / \mathrm{dL}$; 95\% CI, 148-208 in survivors vs. $129 \mathrm{mg} / \mathrm{dL}$; 95\% CI, 79-179 in nonsurvivors, $p=.095)$. Neither mean glucose, its SD, or Mean Amplitude of Glycemic Excursions during the glucose recording differed between survivors and nonsurvivors.

Table 2 summarizes the main patient characteristics in each group. Representative examples of glycemic profiles from
Table 2. Differences between survivors and nonsurvivors

\begin{tabular}{|c|c|c|c|}
\hline & $\begin{array}{c}\text { Survivors } \\
\mathrm{n}=28\end{array}$ & $\begin{array}{c}\text { Nonsurvivors } \\
n=10\end{array}$ & $p$ \\
\hline 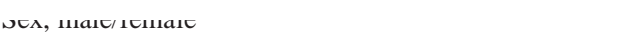 & $1 / 111$ & $\mathrm{U} /<$ & 策 \\
\hline Age, yrs (95\% CI) & $57(52-63)$ & $62(50-73)$ & $.42(\mathrm{NS})^{b}$ \\
\hline History of diabetes mellitus type $2, \mathrm{n}$ (\% of patients) & $10(36)$ & $1(10)$ & .22 (NS) $^{a}$ \\
\hline APACHE II score at first 24 hrs $(95 \%$ CI) & $21(19-23)$ & $19(15-22)$ & $.30(\mathrm{NS})^{b}$ \\
\hline SAPS 3 score $(95 \%$ CI) & $60(55-64)$ & $65(57-73)$ & $.27(\mathrm{NS})^{b}$ \\
\hline PCR (95\% CI) & $117(77-157)$ & $106(39-174)$ & .79 (NS) $^{b}$ \\
\hline Glucose at admission, mg/dL (95\% CI) & $178(148-208)$ & 129 (79-179) & $.095(\mathrm{NS})^{b}$ \\
\hline \multicolumn{4}{|l|}{ Daily insulin during the registry } \\
\hline n (\% of patients) & $17(61)$ & $7(70)$ & \\
\hline Mean dose, IU & 35 & 36 & \\
\hline Median glucose during the registry, mg/dL (95\% CI) & $138(124-151)$ & $144(122-167)$ & $.60(\mathrm{NS})^{b}$ \\
\hline SD of glucose during the registry, mg/dL (95\% CI) & $17(13-20)$ & $22(16-29)$ & $.13(\mathrm{NS})^{b}$ \\
\hline MAGE $(95 \% \mathrm{CI})$ & $41(30-51)$ & $34(17-51)$ & .5 (NS) $^{b}$ \\
\hline DFA $(95 \% \mathrm{CI})$ & $1.49(1.44-1.53)$ & $1.60(1.52-1.68)$ & $.015^{b}$ \\
\hline
\end{tabular}

CI, confidence interval; APACHE, Acute Physiology and Chronic Health Evaluation; SAPS, Simplified Acute Physiology Score; PCR, polymerase chain reaction; MAGE, Mean Amplitude of Glycemic Excursions; DFA, detrended fluctuation analysis.

${ }^{a}$ Fisher's exact test; ${ }^{b}$ analysis of variance. The $p$ values refer to the differences between the groups.
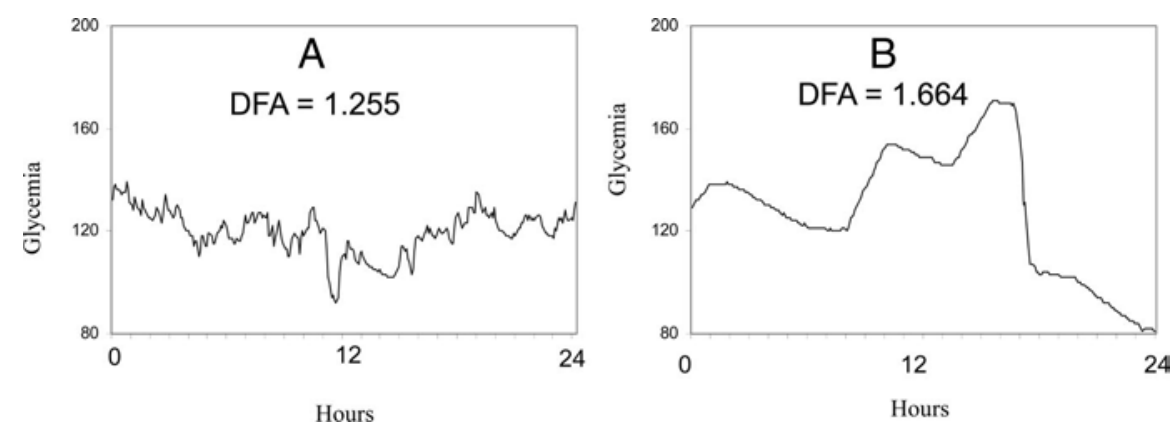

Figure 1. Examples of glycemic profile. Two examples of glycemic curves, from a survivor $(A)$ and a nonsurvivor $(B)$. Although the glycemic values are not significantly different (average glycemia, 119 $\mathrm{mg} / \mathrm{dL}$ in $A ; 128 \mathrm{mg} / \mathrm{dL}$ in $B$ ), the complexity of the survivor's profile $A$ is greater (lower detrended fluctuation analysis $[D F A])$ than that of the nonsurvivor.

a surviving and a nonsurviving patient are shown in Figure 1.

\section{Complexity Analysis}

DFA was significantly lower (indicating higher complexity) in surviving patients than in patients dying in the ICU (1.49 [95\% CI, $1.44-1.53$ ] vs. 1.60 [95\% CI, 1.52-1.68], $F(1,36)=6.548, p=$ .015). This difference persisted even after inclusion of the presence of diabetes, age, APACHE II, and SAPS 3 score in the model $(F(1,31)=12.581, p=.001)$.

DFA did not differ according to gender, and did not correlate with age, APACHE II, or SAPS 3 scores. We found no significant differences in DFA in relation to type of feeding (oral, enteral, or parenteral) or in relation to the amount of insulin administered during the registry.

There was a positive significant correlation between DFA and both mean glucose $(r=.339, p=.046)$ and glucose SD
(Spearman's $g=0.457, p=.006$ ) during the testing day. There was no correlation between Mean Amplitude of Glycemic Excursions and DFA.

DFA was not different between diabetic and nondiabetic patients (1.51 vs. $1.52, p=.92)$. However, survival acted as a confounding factor, and when controlling for this variable, the DFA estimated marginal mean for diabetic patients was 1.67 (95\% CI, $1.60-1.79)$ vs. 1.54 (95\% CI, 1.49-1.59) in nondiabetic patients ( $p=.039$ for diabetes, $p=.001$ for survival, $p=.025$ for the interaction diabetes $\times$ survival).

In a logistic regression model, DFA was significantly associated with mortality (-2LLR, 37.729, $p=.028)$. The odds ratio for death associated with a 0.1 increase in DFA was 2.18 (95\% CI, 1.094.37). These results did not change significantly after controlling for the presence of diabetes (-2LLR, 33.984, $p=$ 
.019 ; odds ratio, 2.53; 95\% CI, $1.16-$ 5.49).

\section{DISCUSSION}

Our data show a significant difference in the complexity of the glycemic profile between nonsurvivors and survivors in a multidisciplinary ICU patient population. Loss of complexity in the glycemic profile is accompanied by a higher odds ratio of death, and the size of this effect is not negligible: a 0.1 increase in DFA roughly doubles the odds of death for the patient.

An unexpected finding was the lack of differences in DFA between diabetic and nondiabetic patients, because other studies $(23,24)$ have consistently found an increased DFA in diabetes. However, our results are probably due to confounding factors. The odds of survival were paradoxically increased in diabetic patients (10:1 vs. 18:9), and when this variable was controlled, a clear difference in estimated marginal means appeared.

Dynamic measures of glucose time series seem to provide complementary-and perhaps more powerful-information on glucoregulation than conventional glycemic analysis. These measurements differ from traditional variability measurements because they are not related to the magnitude of variability, but rather to its organization. Our hypothesis is that a healthy regulatory system is able to detect small changes in glucose concentration and makes continuous small adjustments. Even minor changes in glucose concentration might launch prompt counterregulatory measures to adjust the glucose concentration. Thus, the tracing of glucose concentration would be characterized by frequent small ups-and-downs displaying high complexity (low DFA) and low variability (low SD). A failing regulatory system may require bigger changes in glucose concentration to launch a counterregulatory response. In this case, the tracing of glucose concentration would show low complexity (high DFA) and high variability (high SD). As expected, our sample displayed an inverse correlation between complexity and variability (direct correlation between DFA and SD of the glycemic tracing: Spearman's $\mathrm{g}=$ 0.457, $p=.006$ ).

It has been suggested that scale invariance may be a central organizing principle of physiologic structure and function. The diminished complexity (i.e., in- creased DFA) of the glucose profile in nonsurvivors suggests a breakdown of this scale invariant, fractal organization, and, thus, a failing glucoregulatory system, even before the development of overt hyperglycemia.

To our knowledge, this is the first attempt to quantify the complexity of glucose profile in critically ill patients. Ogata et al $(23,31)$ analyzed diabetes-related alterations of glucose control by means of DFA by use of a CSMG, reporting a mean DFA 1.25 】 0.29 in healthy volunteers. All of the critically ill patients in our series had DFA values greater than those reported by Ogata et al $(23,31)$, which supports our hypothesis that critical illness and multisystem organ dysfunction are characterized by a loss of complexity in the glycemic profile.

An obvious limitation of our study is that the accuracy of point-of-care blood glucose testing in critically ill patients has proven to be poor $(32,33)$. Our measurements are based on a subcutaneous CGMS. Corstjens et al (34) have studied the accuracy of this method in critically ill patients, and their results show a Pearson correlation coefficient of 0.89 for CGMS vs. blood gas analyzer. On the other hand, it is worth noting that an advantage of dynamic methods is that, rather than aiming at the specific value of each measure, they focus on how each measure relates with previous and successive ones. They are more interested in variation than in values, and this makes them more robust against systematic bias.

Our recruiting rhythm was admittedly slow. We had to share the CGMS device with the Departments of Endocrinology and Pediatrics and, naturally, patient caring was a priority. Thus, the studied patients constituted a convenience sample, not a consecutive series of patients admitted to the ICU, and we could not maintain standardized timing of complexity measurements. In all patients, the glycemic profile was obtained during their first week of ICU stay (four patients on day $1 ; 15$ patients on day 2; 11 patients on day 3; two patients on day 4; four patients on day 5; and two patients on day 6). In two cases, the initial continuous glucose series failed, and had to be repeated. For technical reasons, this was not possible until weeks 2 and 3, respectively. Both patients were survivors. The exclusion of these cases from the analysis did not change the results (i.e., DFA sur- vivors, 1.48; nonsurvivors, $1.60 ; p=$ .018) .

Another limitation of our study is that patients were not following a standardized protocol for feeding, infusions, and insulin administration. Nevertheless, in acutely ill patients admitted to an ICU, these types of rigid protocols are probably not feasible, and we were willing to reflect a real-life situation.

As for the mechanisms underlying this loss of complexity, one can only speculate. A seductive explanation could be based on the "uncoupling" often observed in failing complex systems. Another obvious candidate is insulin resistance in a context of increased counterregulatory hormones. A more unlikely and less physiologically attractive (but perhaps more clinically useful) hypothesis would assume that the impaired complexity in the interstitial fluid only represents a poor hemodynamic status, with peripheral hypoperfussion and delayed equilibrium.

As in previous studies, we do not establish whether glycemic dysregulation is the cause of an impaired prognosis or whether it is just a marker of physiologic breakdown. However, our results indicate that complexity analysis may constitute a powerful tool in the study of glycemic control in critical care patients.

\section{CONCLUSIONS}

In critically ill patients, there is a difference in the complexity of the glycemic profile between survivors and nonsurvivors. Loss of complexity in glycemia time series, evaluated by DFA, correlates with higher mortality. Complexity analysis may offer new insights in issues involving glucose control in critically ill patients.

\section{ACKNOWLEDGMENTS}

We thank Nieves Franco, Gemma García-Navaza, Rosa Asensio, Margarita Mas, Silvia Hernandez, Ignacio Temprano, and Alberto Orejas for their help in data retrieval, and Sandra Oltra and Pau Miro for their assistance.

\section{REFERENCES}

1. McCowen KC, Malhotra A, Bistrian BR: Stress-induced hyperglycemia. Crit Care Clin 2001; 17:107-124 
2. Dungan KM, Braithwaite SS, Preiser J: Stress hyperglycaemia. Lancet 2009; 373: 1798-1807

3. Saberi F, Heyland D, Lam M, et al: Prevalence, incidence, and clinical resolution of insulin resistance in critically ill patients: An observational study. J Parenter Enteral Nutr 2008; 32:227-235

4. Yendamuri S, Fulda GJ, Tinkoff GH: Admission hyperglycemia as a prognostic indicator in trauma. J Trauma 2003; 55:33-38

5. Krinsley JS: Association between hyperglycemia and increased hospital mortality in a heterogeneous population of critically ill patients. Mayo Clin Proc 2003; 78: 1471-1478

6. Freire AX, Bridges L, Umpierrez GE, et al: Admission hyperglycemia and other risk factors as predictors of hospital mortality in a medical ICU. Chest 2005; 128:3109-3116

7. van den Berghe G, Wouters P, Weekers F, et al: Intensive insulin therapy in the critically ill patients. $N$ Engl J Med 2001; 345: 1359-1367

8. Wiener RS, Wiener DC, Larson RJ: Benefits and risks of tight glucose control in critically ill adults: A meta-analysis. JAMA 2008; 300: 933-944

9. NICE-SUGAR Study Investigators, Finfer S, Chittock DR, et al: Intensive versus conventional glucose control in critically ill patients. $N$ Engl J Med 2009; 360: 1283-1297

10. Egi M, Bellomo R, Stachowski E, et al: Variability of blood glucose concentration and short-term mortality in critically ill patients. Anesthesiology 2006; 105:244-252

11. Krinsley JS: Glycemic variability: A strong independent predictor of mortality in critically ill patients. Crit Care Med 2008; 36: 3008-3013

12. Service FJ, Molnar GD, Rosevear JW, et al: Mean amplitude of glycemic excursions, a measure of diabetic instability. Diabetes 1970; 19:644-655

13. Pikkujämsä SM, Mäkikallio TH, Sourander LB, et al: Cardiac interbeat interval dynamics from childhood to senescence: Comparison of conventional and new measures based on fractals and chaos theory. Circulation 1999; 100:393-399

14. Vikman S, Makikallio TH, Yli-Mayry S, et al: Altered complexity and correlation properties of R-R interval dynamics before the spontaneous onset of paroxysmal atrial fibrillation. Circulation 1990; 100:2079-2084

15. Makikallio TH, Seppanen T, Airaksinen KE, et al: Dynamic analysis of heart rate may predict subsequent ventricular tachycardia after myocardial infarction. Am J Cardiol 1997; 80:779-783

16. Hornery R, Aboy M, Abasolo D, et al: Complexity analysis of intracranial hypertension using approximate entropy. Crit Care Med 2006; 34:87-95

17. Toweill D, Sonnenthal K, Kimberly B, et al:
Linear and nonlinear analysis of hemodynamic signals during sepsis and septic shock. Crit Care Med 2000; 28:2051-2057

18. Papaioannou VE, Maglaveras N, Houvarda I, et al: Investigation of altered heart rate variability, nonlinear properties of heart rate signals, and organ dysfunction longitudinally over time in intensive care unit patients. J Crit Care 2006; 21:95-103

19. Varela M, Calvo M, Chana M, et al: Clinical implications of temperature curve complexity in critically ill patients. Crit Care Med 2005; 33:2764-2771

20. Varela M, Churruca J, Gonzalez A, et al: Temperature curve complexity predicts survival in critically ill patients. Am J Respir Crit Care Med 2006; 174:290-298

21. Streletz LJ, Reyes PF, Zalewska M, et al: Computer analysis of EEG activity in dementia of the Alzheimer's type and Huntington's disease. Neurobiol Aging 1990; 11:15-20

22. Bylsma FW, Peyser CE, Folstein SE, et al: EEG power spectra in Huntington's disease: Clinical and neuropsychological correlates. Neuropsychologia 1994; 32:137-150

23. Ogata H, Tokuyama K, Nagasaka S, et al: Long-range negative correlation of glucose dynamics in humans and its breakdown in diabetes mellitus. Am J Physiol Regul Integr Comp Physiol 2006; 291:R1638R1643

24. Churruca J, Vigil L, Luna E, et al: The route to diabetes: Loss of complexity in the glycemic profile from health through the metabolic syndrome to type 2 diabetes. Diabetes, Metabolic Syndrome and Obesity 2008; 1:3-11

25. Goldstein B, Fisher DH, Kelly MM, et al: Decomplexification in critical illness and injury: Relationship between heart rate variability, severity of illness, and outcome. Crit Care Med 1998; 26:352-357

26. Kennedy HL: Heart rate variability-A potential, non-invasive prognostic index in the critically ill patient. Crit Care Med 1998; 26: 213-214

27. Seely AJ, Christou NV: Multiple organ dysfunction syndrome: exploring the paradigm of complex nonlinear systems. Crit Care Med 2000; 28:2193-2200

28. Rebrin K, Steil GM: Can interstitial glucose assessment replace blood glucose measurements? Diabetes Technol Ther 2000; 2:461-472

29. Konschinsky T, Heinemann L: Sensors for glucose monitoring: technical and clinical aspects. Diabetes Metab Res Rev 2001; 17: 113-123

30. Peng CK, Havlin S, Stanley HE, et al: Quantification of scaling exponents and crossover phenomena in nonstationary heartbeat time series. Chaos 1995; 5:82-87

31. Ogata H, Tokuyama K, Nagasaka S, et al: Long-range correlated glucose fluctuations in diabetes. Methods Inf Med 2007; 46: 222-226

32. Louie RF, Tang Z, Sutton DV, et al: Point-ofcare glucose testing: Effects of critical care variables, influence of reference instruments, and a modular glucose meter design. Arch Pathol Lab Med 2000; 124:257-266

33. Scott MG, Bruns DE, Boyd JC, et al: Tight glucose control in the intensive care unit: Are glucose meters up to the task? Clin Chem 2009; 55:18-20

34. Corstjens AN, Ligtenberg JJ, van der Horst IC, et al: Accuracy and feasibility of point-ofcare and continuous blood glucose analysis in critically ill ICU patients. Crit Care 2006; 10:R135

\section{Appendix}

\section{Detrended Fluctuation Analysis}

Detrended fluctuation analysis (DFA) attempts to disclose patterns of self-similarity in time plots. In other words, it looks for the presence of "memory" in the curve, understood as long-range correlations.

To be able to perform DFA, it is first necessary to integrate the time series:

$$
y(k)={ }_{i=1}^{k}\left(G_{i}-G_{\text {mean }}\right)
$$

(Fig. 2A), where $G_{\mathrm{i}}$ is each individual point, and $G_{\text {mean }}$ is the mean of the series as a whole.

Next, the integrated curve is divided into time segments of size $n$ (Fig. 2, $B-D$ ). A regression line is calculated for each segment, and the difference between the integrated curve and the different regression lines is computed as:

$$
F(n)=\longdiv { \overline { 1 } _ { k = 1 } ^ { N } [ y ( k ) - y _ { n } ( k ) ] ^ { 2 } , }
$$

where $F(n)$ is the area between the integrated curve and the regression lines, $\mathrm{N}$ is the total number of data points, $y(k)$ is the value of the integrated curve at each point, and $y_{n}(k)$ is the value of the regression line at that point.

This operation is repeated for different time frames (that is, for different values of $n$ ). The smaller the time scale ( $n$ ), the better the fit of the regression lines to the integrated curve and the lower the value of $F(n)$. Conversely, the value of $F(n)$ tends to increase exponentially as the time frame $(n)$ increases.

Finally, the relation between $F(n)$ and the size of $n$ is analyzed. A plot is drawn with $\log [F(n)]$ on the $y$-axis and $\log (n)$ on the $x$-axis (Fig. 2E). A good fit to a regression line indicates the existence of scaling (self-similarity), and a fractal structure can be assumed. 

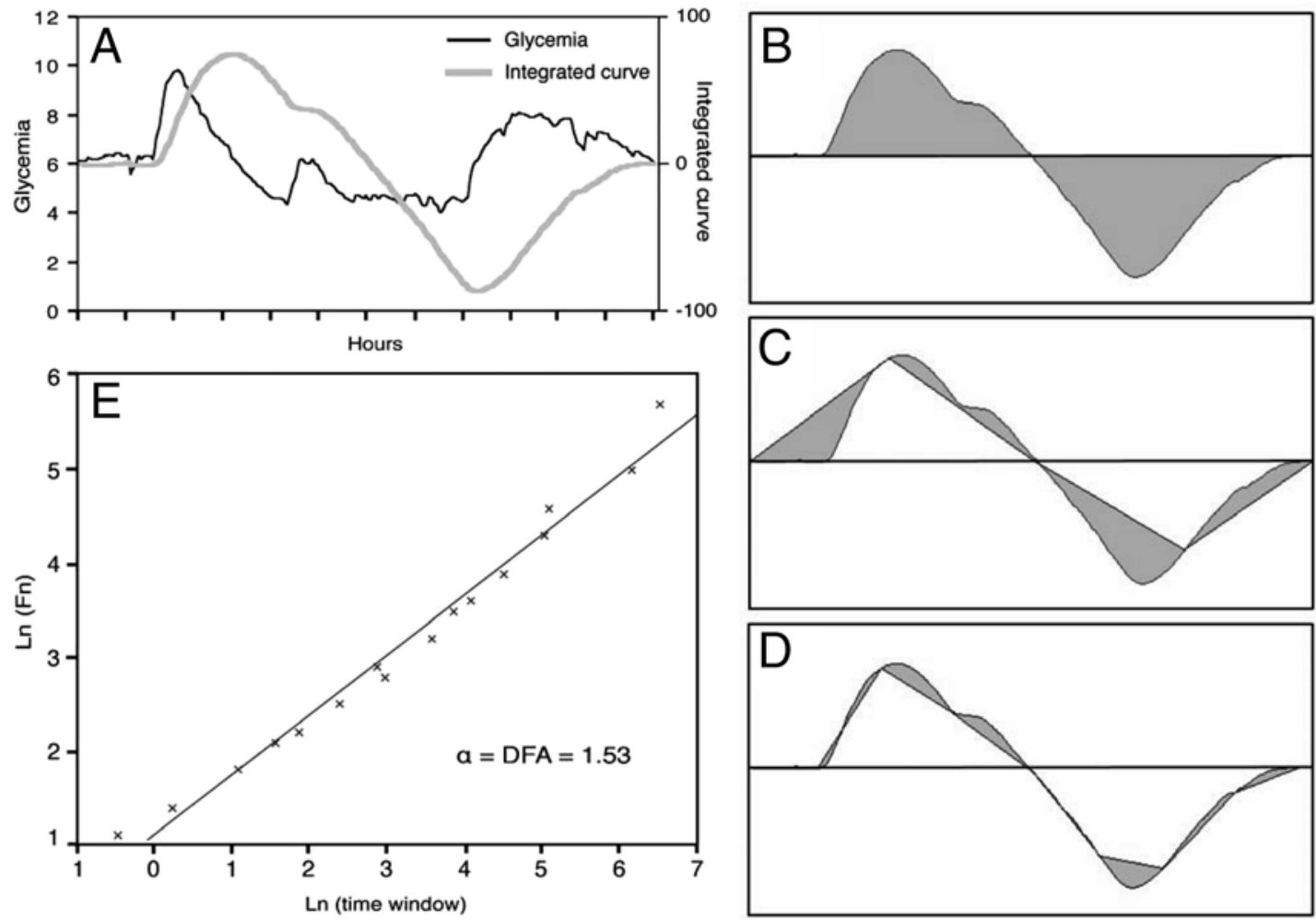

Figure 2. Detrended fluctuation analysis (DFA). From the original time series, an integrated curve is obtained $(A)$.

$$
y(k)={ }_{i=1}^{k}\left(G_{\mathrm{i}}-G_{\text {mean }}\right),
$$

where $G_{\mathrm{i}}$ indicates each individual point; $G_{\text {mean }}$ indicates mean of the series as a whole. This integrated curve will be utilized for further calculations. The integrated curve is divided into progressively smaller time segments $(B, C, D)$. A regression line is calculated for each segment, and the total difference between the integrated curve and the regression lines is calculated for each time window $(F(n)$,gray area). The smaller the time window, the better the fit of the regression line and the lower the value of $F(n)$. Finally, a plot is drawn $(E)$ with $\log (F(n)$ in the y-axis and log (time-window) in the x-axis. A good fit reveals the presence of scaling (self-similarity). DFA is the slope of the regression line. It displays the scaling exponent and is an indicator of the degree of complexity of the curve.

DFA is the slope of the regression line (a). It displays the scaling exponent, and is an indicator of the degree of complexity of the curve. In an entirely random time series ("white noise”), $a=0.5$. A $1 / \mathrm{f}$ type time series will have $a=1$. A "random walk" (the integration of a random series, "brown noise") will display $\mathbf{a}=1.5$. Long-range negatively correlated fluctuations will show a $<1.5$, whereas in positive correlations, $a>1.5$.

On the whole, a curve is more complex (less predictable) the closer its value of $a$ is to 0.5 . (Values of $a<0.5$ reveal anticorrelations, which also implies a certain degree of predictability, and hence, a lower level of complexity).

In our series, $\mathrm{n}=288$.

The program used to calculate DFA was written in Python (http://www. python.org) and is available from the authors on request. 